Revista de Derecho

\title{
Subjetividad de las máquinas en toma de decisiones judiciales
}

\author{
Subjectivity of Machines \\ in Judicial Decision-Making
}

Cristian Fernando Montañez Sierra

Investigador independiente

Bogotá D.C., Colombia

crisfermon@gmail.com

ORCID: 0000-0003-1112-882X

Artículo de investigación

DOI: https://doi.org/10.32719/26312484.2021.36.1

Fecha de recepción: 16 de octubre de 2020

Fecha de revisión: 11 de noviembre de 2020

Fecha de aceptación: 16 de marzo de 2021

Fecha de publicación: 1 de julio de 2021 


\section{RESUMEN}

El cuestionamiento de la subjetividad del agente legal que, ejecutando sus labores y basada en discrecionalidad judicial imprime aspectos particulares como emociones, propios de la esencia humana en la sentencia, es el tema central de este artículo. Con la implementación de nuevos actores como jueces digitales, estos aspectos adquieren un papel relevante por quien ahora se encarga de deliberar.

El objetivo del artículo es evaluar el papel de la subjetividad, inicialmente desde el rol de jueces y/o juezas, para luego involucrar programadores y programadoras de inteligencia artificial que asisten tareas en la toma de decisiones judiciales. ¿Qué pasa con la subjetividad que ya no impregna directamente el encargado de juzgar? En otras palabras, la empatía que genera en la actualidad el agente judicial encuentra nuevos aliados con quienes trabajan mancomunadamente.

El análisis está basado en artículos que profundizan temas como decisiones judiciales, emociones e inteligencia artificial. Temáticas trabajadas desde conocedores de ciencias como derecho y tecnología que unifican esfuerzos para dar origen a conocimiento superior artificial. Al procesar grandes volúmenes de información emanan una sentencia, que en vector doctrinal anhela mantener certeza jurídica y cumplir con buenas prácticas que ocasionen en la sociedad aceptación y legitimidad.

PALABRAS CLAVE: decisiones, empatía, emociones, ética, inteligencia, jueces, responsabilidad, subjetividad.

\section{ABSTRACT}

The questioning of the subjectivity of the legal agent who, executing their work and based on judicial discretion, prints particular aspects such as emotions, typical of the human essence in the sentence, is the central theme of this article. With the implementation of new actors such as digital judges, these aspects acquire a relevant role for those who are now in charge of deliberating.

The objective of the article is to evaluate the role of subjectivity, initially from the role of judges, to later involve artificial intelligence programmers who assist tasks in making judicial decisions. What about the subjectivity that would no longer directly permeate the one in charge of judging? In other words, the empathy that the judicial agent currently generates finds new allies with whom they work together.

The analysis is based on articles that deepen into issues such as judicial decisions, emotions and artificial intelligence. Topics worked from connoisseurs of sciences such as law and technology that unify efforts to give rise to artificial superior knowledge. When processing large volumes of information, they emanate a sentence, which as a doctrinal vector wishes to maintain legal certainty and comply with good practices that cause acceptance and legitimacy in society.

KEYWORDs: decisions, empathy, emotions, ethics, intelligence, judges, responsibility, subjectivity. 


\section{INTRODUCCIÓN}

¿A lguna vez se ha cuestionado sobre la subjetividad de las máquinas cuando el enA cargado de juzgar no es un ser humano? Esta es la inquietud que ha promovido el desarrollo del artículo académico que se expondrá a continuación. En noviembre de 2019 , se expuso en un seminario de inteligencia artificial, ${ }^{1}$ que la subjetividad suprimida del juez o jueza estaría determinada por el programador o programadora, quien en ejercicio de sus actividades tendría la misión de crear y educar al sistema de aprendizaje automático o machine learning ${ }^{2}$ encargado de decidir.

El cuestionamiento central corresponde al dilema expresado en el párrafo anterior, interrogado de la siguiente manera: ¿Qué ocurre con la subjetividad suprimida de la autoridad judicial al momento de emitir decisiones judiciales, asistida ahora por el aprendizaje automático y cuyo elemento puede ser impreso por el/la programador(a) informático(a)? Inicialmente puede decirse que, "[c]iertamente los grandes logros en los agentes artificiales que buscan una inteligencia artificial superior a la humana están encabezados por agentes que no exhiben la facultad de experimentar emociones o sentimientos".

Explorar la autonomía de la autoridad judicial, quien por su carácter de humano comprende habilidades, sentimientos, emociones y sesgos, permite afrontar elecciones de bueno y malo, entre la búsqueda racional de objetividad enfrentándose a la dualidad con la subjetividad. Aplicando inteligencia artificial este tema puede darse por superado. El aprendizaje automático, base de los jueces digitales ${ }^{4} \mathrm{y}$ las decisiones judiciales asistidas, ${ }^{5}$ pregona un cien por ciento de objetividad en el acto adjudicativo. ¿Esto es real?

1. Daniel Peña Valenzuela, "Inteligencia artificial y derecho" (Seminario IV: Inteligencia artificial, Universidad Externado de Colombia, 2019).

2. IBM, "Machine Learning", 22 de junio de 2020, https://ibm.com/ar-es/analytics/machine-learning, párr. 1. "Machine learning es una forma de la Inteligencia Artificial que permite a un sistema aprender de los datos en lugar de aprender mediante la programación explícita. Sin embargo, machine learning no es un proceso sencillo. Conforme el algoritmo ingiere datos de entrenamiento, es posible producir modelos más precisos basados en datos. Un modelo de machine learning es la salida de información que se genera cuando entrena su algoritmo [...] Después del entrenamiento, al proporcionar un modelo con una entrada, se le dará una salida".

3. Antonio Rico Sulayes, "Hacia el reconocimiento de la subjetividad en los agentes artificiales. Una delimitación del sujeto artificial en la semiótica", Tópicos del Seminario 41 (2019): 163-4.

4. Belén Campos, "China y Estonia implantan jueces virtuales para acelerar procesos", El cierre digital: Investigación dirigida a la verdad, 14 de octubre de 2019, https://elcierredigital.com/sucesos/973432190/ jueces-virtuales-china-estonia-implantan-algoritmos-agilizan-trabajo-judicial.html.

5. Cristian Fernando Montañez Sierra, "Decisiones judiciales asistidas: Paradigmas del juez y jueza en cuanto al uso de inteligencia artificial" (tesis de maestría de próxima aparición, Universidad Andina Simón Bolívar, Sede Ecuador, s. f.). 
Identificados los actores principales en el proceso dictatorial, es necesario - desde la perspectiva del investigador - diferenciar el mundo de la empatía humana. Los individuos, a partir de vínculos sociales, construyen redes en las cuales sesgos y subjetividades están presentes en sus acciones, por ende, revisar estos elementos desde los protagonistas, permitirá orientar el proceso de enseñanza de sistemas de aprendizaje automático, y empezar a vislumbrar propuestas desde conceptos, autores y complementos involucrados.

Las emociones pueden considerarse punto neurálgico al momento de decidir. El juez se enfrenta a una solución basada en intelecto, acompañada de voluntad. Como resultado, la subjetividad puede ser un factor que permea dicha deliberación generando cuestionamientos en la sentencia, y donde esta discrecionalidad como principio rector cobija a jueces y juezas en el desarrollo de sus actividades, pero no exime la aparición de características propias e individuales que emergen en medio de los procesos.

Es probable que la aplicación de la ética como disciplina permita encontrar un punto intermedio entre las dos subjetividades. La inteligencia artificial como herramienta de apoyo, asistencia, ayuda o auxiliar despliega sus componentes en favor de realizar actividades en las cuales se considera elemental la intervención de personas y está no se desplazará, encontrando en catálogos axiológicos y el principio de responsabilidad, aliados vitales para la ejecución efectiva de conductas sociales aceptadas, que mantienen el equilibrio comunal, previenen el daño y son necesarias en el uso de inteligencia artificial.

Bienvenidos a un recorrido teórico-conceptual sobre los nuevos entes encargados de decidir, donde el ser humano sigue siendo un actor determinante al momento de revisar fallos obtenidos por jueces digitales, identificando la subjetividad como factor interviniente desde el rol de juzgador y programador, pero entendiendo como emociones, sesgos, ética y responsabilidad se encuentran en medio de dos postulados fundamentales para el proceso judicial basado en tecnología.

\section{EMPATÍA DE LA AUTORIDAD JUDICIAL: SESGOS Y SUBJETIVIDAD}

La naturaleza humana refleja un innumerable conjunto de condiciones, en las que su relación con el prójimo permite encontrar similitudes o diferencias que influyen en su autenticidad y trato. Como punto de partida, es deseo del investigador ilustrar la concepción de aquellas características naturales, inherentes a la persona, de manera especial, como componentes determinantes al momento de tomar decisiones, ejecutar acciones y asumir consecuencias. ¿Qué conocemos como empatía humana? ¿A qué hace referencia? 
El término fue acuñado hacia el año 1909, "se construyó de una raíz griega que significa emoción y pasión", ${ }^{6}$ es decir que, este concepto se compone de elementos que ayudan en cuanto a la apropiación de hechos se refiere. "Se entiende como la capacidad (o el fenómeno) de sentir como propias emociones ajenas, o sea, la capacidad de ponerse en los zapatos del otro, lo que ha sido reconocido por muchos autores como el origen de la moral". ${ }^{7} \mathrm{Y}$ tal como menciona el autor, se relaciona con el pensamiento expresado por el economista y filósofo escocés Adam Smith en el libro La teoría de los sentimientos morales. ${ }^{8}$

La empatía hace parte del entorno global de los seres vivos, no puede atribuirse exclusivamente a los individuos, es posible verlo reflejado en las acciones de diferentes seres que, en el ejercicio de las emociones y sentimientos expresan percepciones basadas en la apreciación del otro. El estudio de esta especificidad de comportamientos ha demostrado que "se localizó con precisión el lugar del cerebro donde ocurre"; 9 por tanto, la dicotomía entre razón y deseo se ve expresada en un proceso desde una ubicación física puntual.

Las afinidades (o no) que experimentan las personas — simpatía, cercanía, proximidad, antipatía, ecpatía ${ }^{10} \mathrm{o}$ indiferencia- involucran estructuras propias que ocasionan el procesamiento cerebral; por tanto:

Según la neurociencia, hay tres componentes en la empatía humana. El primero es el emocional [...]. Un segundo componente es cognitivo, la capacidad de entender racionalmente las causas y condiciones de los sentimientos del otro. Finalmente hay un componente de compasión, que es el que motiva a actuar para aliviar los sufrimientos. ${ }^{11}$

Como resultado, tanto el encargado de juzgar como de programar tienen inmersas estas concepciones en su ser intelectivo y volitivo. "No debe olvidar la empatía para percibir con certeza, las necesidades de quien requiere justicia"; ${ }^{12}$ de allí que, el

6. Moisés Wasserman, "Empatía", ProQuest, 24 de enero de 2020, https://proquest.com/docview/23441441 41 ? accountid $=8308$.

7. Ibíd.

8. Adam Smith y Carlos Rodríguez Braun, La teoría de los sentimientos morales (Madrid: Alianza, 1997).

9. Wasserman, "Empatía".

10. José Luis González de Rivera y Revuelta, "Empatía y ecpatía”, Psiquis 25, n. 6 (2004): 243. Básicamente, es la estrategia de equilibrio establecida para protegerse a si mismo de las consecuencias provocadas por la "fatiga por compasión", síndrome que aparece cuando nos concentramos solo en las emociones del otro sin tomar en cuenta las propias; es decir, vivir los problemas, las tristezas o la baja autoestima ajena y permitir espacios en los que nos afecten como si fueran nuestras.

11. Ibíd.

12. Graciela Noelia Herbas, "La sensibilidad humana en el profesional del derecho como instrumento esencial para una efectiva justicia”, Revista Derecho y Salud (2018): 98. 
contraste de la subjetividad, empatía y emociones empieza a ser preponderante en la evaluación sobre su aplicabilidad en el derecho y las decisiones judiciales, sobre todo porque si quien decide es una herramienta de inteligencia artificial, carecería de estos elementos, aunque revestida de objetividad en su mayor esplendor, ¿esto es así?

Parte del deterioro de las sociedades modernas se atribuye a una crisis de valores que desbordan los índices de discriminación, desigualdad y exclusión. El catálogo axiológico que los contiene se basa en el desarrollo subjetivo de cada individuo, sesga su visión y delimita en contextos propios y ajenos, de manera que su determinación se ve influenciada por lo que cree justo, correcto y verdadero. Por consiguiente, "para Kant la realidad solo existe en tanto hay sujetos que la conocen y le dan forma; es el paso de la moral subjetiva", ${ }^{13}$ por eso, ¿qué ocurre con la inteligencia artificial y la subjetividad personal?

Entonces, un sujeto es libre, sí, pero en sentido negativo. Todo aquello que hay alrededor del sujeto, lo limita, lo determina, lo niega. La libertad en sentido positivo es la absoluta determinación y limitación de un ser por sí mismo, y esto realmente no llega a suceder del todo. La individualidad del sujeto es una afirmación parcial, porque esta afirmación, si bien puede entenderse como que cada sujeto consiste en sí mismo, sin embargo, las ideas, deseos, normas y preferencias le llegan a los sujetos, mayoritariamente, impuestos por su entorno social. ${ }^{14}$

Frente al cuestionamiento anterior, la inteligencia artificial no posee subjetividad, por ende, empatía, emociones o sentimientos. Se puede decir que entre obligación y convicción se genera un nuevo encuentro de conocimiento. La obligación como regla sujeta a revisión de alguien se contrapone a la convicción, cuya determinación está dada por un grado de conciencia y entendimiento superior. Entonces, los actores intervinientes en el proceso de decisiones judiciales asistidas pueden hablar de empatía, basada en la obligación de hacer lo correcto porque así lo determina la ley y buenas prácticas, y a su vez, los dos persiguen convicciones dadas desde su aprendizaje.

Comprender la perspectiva de la situación, puede llamarse clave en medio del proceso de creación y educación entre abogados y programadores. Su empatía es única, el aprendizaje automático per se no entiende de rasgos personales, pero puede verse impregnado en actividades que conlleven la formación de entes tecnológicos en ciencia jurídica. Sigue siendo el ser humano el actor principal. No importa quién cree in-

13. Jesús Víctor Alfredo Contreras Ugarte, "Cimentando en valores para empoderar en derechos y al derecho. Empatía del reconocimiento. Una reflexión desde la filosofía de Hegel”, Universitas. Revista de Filosofía, Derecho y Politica, n. 23 (2016): 168.

14. Ibíd., 171. 
novaciones tecnológicas y cual sea su fin, está en el trabajo de reconocimiento actuar en beneficio de los sistemas expertos que servirán a la humanidad.

El rol del juez o jueza frente a la interpretación y argumentación jurídica, su responsabilidad de hacer sociedad centra esfuerzos en el ejercicio de una función basada en valores humanos propios de formación, intelecto y servicio. Al ser asistida la labor judicial, el ente informático ahora encargado de dirimir el conflicto elimina conductas propias de la humanidad. Sesgo y subjetividad cuestionada en la práctica podrían ser suministrados por el encargado de educar a través del aprendizaje automático.

La interpretación del otro como parte del proceso empático, acompaña ahora la interpretación jurídica. Sin tomar posición es muy probable que el sistema de aprendizaje automático cumpla a cabalidad la segunda anotación. ¿Es necesario que cumpla con la primera? ¿Se necesitan sistemas digitales empáticos? Si bien la idea es aclarar conceptos y acercar al lector a un tema trascendental del derecho en materia de creación de actos adjudicativos, los interrogantes no dejan de aparecer. "El acto empático [...] sostiene que la identidad solo se constituye desde la alteridad". ${ }^{15}$

Educar la empatía como objetivo de evolución solidaria y profesional, constituirá un pilar multidisciplinar y que trae ventajas a individuos. Se ha identificado que existen factores intrínsecos de los intervinientes en el proceso judicial que hace particular el ejercicio de la profesión. "Androides ${ }^{16}$ y ginoides ${ }^{17}$ no serán capaces, por el momento, de comprender con empatía, pero sí pueden llegar a reconocer e interpretar situaciones emocionales y actuar en consecuencia". ${ }^{18}$

Las innovaciones tecnológicas basadas en agentes racionales contemplan, cómo elementos particulares de las personas son suprimidos o impregnados desde nuevos actores, antes no considerados. A continuación, se observará el papel de las emociones, integrantes de la empatía humana.

15. Paulina Monjaraz Fuentes, "Reconstruir la comunidad humana desde la empatía: Medios para generar la paz", Open Insight X, n. ${ }^{\circ} 20$ (2019): 79.

16. Revista de Robots, "Androides, qué es un androide humano y ejemplos", Revista de Robots, 30 de enero de 2020, https://revistaderobots.com/robots-y-robotica/androides-que-es-un-androide-humano-y-ejemplos/. "Por definición un androide es un robot o máquina autómata antropomorfa, que posee una apariencia y características similares a las de los humanos. Son máquinas que, de manera autónoma por su configuración, imitan ciertas acciones o conductas que realiza el hombre".

17. Diccionario Sensagent, "Ginoide", accedido 12 de octubre de 2020, http://diccionario.sensagent.com/Ginoide/es-es/. "Ginoide o fembot es la denominación que se le da a un robot antropomorfo de fisionomía femenina, aunque en el lenguaje coloquial el término androide suele usarse para ambos casos, masculino o femenino".

18. Tomás de Andrés Tripero, "Inteligencia emocional artificial: En las fronteras de la robótica", Universidad Complutense de Madrid, s.f., 2. 


\section{EMOCIONES: PUNTO NEURÁLGICO EN LA DECISIÓN JUDICIAL}

El personaje encargado de juzgar experimenta particularidades de la sociedad que intervienen en su proceso deliberativo, aunque las disposiciones normativas impulsen el encuentro objetivo entre razones y decisiones, el ser humano por condición experimenta alteraciones propias a las reacciones de actos cometidos por semejantes. Así, el juez sigue siendo un ser racional, emotivo y empático con la realidad. En el recorrido de esta sección son tres los cuestionamientos que se plantean como desarrollo y explicación del tema central, la emoción.

Para comenzar, ¿qué son las emociones? Puede decirse que es un proceso interno que evoca voluntad de reacción ante situaciones que transforman la esfera individual de las personas. Se exteriorizan por medio de gestos o señas visibles de la cara o el cuerpo; ${ }^{19}$ "los cambios corporales siguen directamente a la percepción del hecho desencadenante y que nuestra sensación de esos cambios según se van produciendo es la emoción". ${ }^{20}$ La comprensión de la acción encuentra en las emociones el instrumento que materializa el sentir de quien, en medio de un acontecimiento, actúa de manera determinada.

[T]odas implican un pensamiento o una percepción intencionales dirigidos a un objeto, y algún tipo de valoración evaluativa de ese objeto realizada desde el punto de vista personal del propio agente. Esa valoración atribuye significación al objeto en términos del esquema de objetivos y fines del agente. Estas valoraciones no tienen por qué ser lingüísticas, ni siquiera complejas [...] Lo único que se necesita para ello es que las criaturas en cuestión perciban el objeto [...] como algo bueno desde el punto de vista de los deseos y objetivos de ellas mismas. ${ }^{21}$

Las emociones pueden ser entendidas como sensaciones o afectos pasivos, "un cierto tipo de percepción o sentimiento de algo que nos ocurre dentro de nosotros mismos durante un lapso más o menos largo de tiempo". ${ }^{22}$ Por tanto, la definición de este grupo está dada en la identificación de objeto y causa que los genera. Como con-

19. Aude Argouse y María Eugenia Albornoz, eds., Sentimientos y justicia: coordenadas emotivas en la factura de experiencias judiciales, Chile, 1650-1990, Colección Dossiers, 2 (Santiago de Chile: Acto Editores, 2016), 253.

20. William James, “¿Qué es una emoción?”, trad. Elena Gaviria Stewart, Estudios de Psicología, n. 21 (1985): 59.

21. Martha Nussbaum, Las emociones politicas: ¿Por qué el amor es importante para la justicia?, trad. Albino Santos Mosquera (Barcelona: Paidós, 2014), 481.

22. Daniel González Lagier, Emociones, responsabilidad y derecho, Colección Filosofía y derecho (Madrid: Marcial Pons, 2009), 29. 
ductas, las emociones se ligan estrechamente con comportamientos, resultan ser útiles en materia de supervivencia y emanan de estados mentales internos o privados de cada persona. ${ }^{23}$ Desde una concepción de rituales mágicos, estos se ponen "en marcha voluntariamente cuando la situación nos sobrepasa y no podemos enfrentarnos a ella de otra forma".${ }^{24} \mathrm{O}$ sencillamente, como juicios, creencias o evaluaciones, atadas al componente cognitivo. ${ }^{25}$

Razón por la cual, las emociones desarrollan a través de una estructura, modelos que permiten exteriorizar elementos de convicción o "juicio evaluativo, el objeto intencional, los cambios fisiológicos, la sensación, la expresión de la emoción y la tendencia a la acción". ${ }^{26}$ Como reacción al encuentro interior siguen los siguientes pasos, de manera que, cuentan una historia que permite desarrollar el proceso emotivo:

1. Las emociones surgen cuando se evalúan las propiedades de un evento en relación con la satisfacción o frustración de un deseo.

2. Las emociones "se enfocan" hacia dicho evento (el objeto intencional), toman una dirección.

3. Se producen ciertos cambios fisiológicos en el cuerpo del sujeto que se encuentra en una situación emocional.

4. Tales cambios se sienten y producen una agitación anímica, que a veces es experimentada como placentera y, otras veces, como dolorosa.

5. El rostro y el cuerpo del sujeto expresan la emoción.

6. El sujeto se siente impulsado a realizar cierta acción. ${ }^{27}$

En contraste con lo expuesto, ¿cómo influyen en el proceso sentenciador? Concebida como una transformación propia de seres humanos, el juez hace uso de emociones al momento de implementar medidas basadas en derecho sobre un evento específico. Podría suponerse que el aspecto emotivo del encargado de deliberar sentencias cumple con lineamientos individuales, categorizados racionales, estando dirigidos bajo una justificación, coherente con quién es él como persona. Pero sobre todo en la aplicación correcta de la norma, adecuando las acciones al hecho que, normado ante sociedades, tiene un carácter axiológico y moral, adquiriendo relevancia.

Puede decirse que, las emociones se ligan estrechamente con la independencia judicial cuyo principio discrecional toma prelación al momento de emitir un fallo. "Las emociones son estados parcialmente dentro del control del agente", ${ }^{28}$ están presentes,

\footnotetext{
23. Ibíd., 38.

24. Ibíd., 43.

25. Ibíd., 44.

26. Ibíd., 77.

27. Ibíd., 62.

28. Ibíd., 151.
} 
pero a la vez se exige al juez o jueza apartar la implicación de subjetividades cuyos intereses particulares tergiversen la ejecución de funciones analíticas, valorativas y concluyentes.

Las emociones no son malas si se garantiza un ejercicio oportuno y correcto del derecho. La evaluación de estas mismas se dará en la omisión de creencias, contextualización del hecho y por ende de medidas correctivas, dando cumplimiento legal a las normas aplicables en ordenamientos jurídicos garantistas de seguridad jurídica. Si se cumplen criterios como los expuestos, la resolución emitida puede considerarse legítima, desde el ámbito jurídico hasta la aceptación social.

Entonces, las emociones influyen en la decisión judicial de dos maneras, positivamente si se usan para valorar en medio de pasos del debido proceso, su incorporación ayuda a esclarecer los factores del caso. Negativamente, si al ponderar entre regla y sentimiento, este último se impone sin justificación válida. Están adheridas a las normas sociales, son la vara de medición por medio de las cuales se otorgará un valor agregado al proceso o, por el contrario, es el eslabón débil que ocasiona trámites jurídicos carentes de fundamento que realcen y promuevan justicia.

El reproche es el primer factor a ser usado cuando las consideraciones que sustentan la decisión no son recibidas de manera ecuánime y oportuna. "Normas sociales y circunstancias societales específicas dan forma al contenido cognitivo de las emociones". ${ }^{29}$ En el agente judicial, la conducta emotiva tiende a ser considerada imparcial, está a su cargo hacer uso del derecho como ciencia jurídica, y donde sus actos se construyen desde una capacidad intelectiva y volitiva, así que, se espera un tratamiento legal acorde con la situación y donde la impregnación de subjetividades sea mínima, por no decir nula.

De modo que, un último interrogante se plantea, ¿qué pasa con los modelos de aprendizaje automático encargados de decidir? Se ha expresado que los afectos propios de los humanos no pueden ser sentidos por algoritmos ${ }^{30}$ encargados de juzgar; pero sí pueden ser simulados. Los gestos como expresión pueden ser tenidos en cuenta en la programación de los modelos de aprendizaje automático. El día que puedan ser interpretados, la decisión judicial podrá ser entendida similar a la manera en que hoy

29. Martha Nussbaum, La ira y el perdón: Resentimiento, generosidad, justicia, trad. Víctor Altamirano (Ciudad de México: Fondo de Cultura Económica, 2018), 332.

30. María Estela Raffino, "Algoritmo en informática", Concepto.de, accedido 12 de octubre de 2020, https:// concepto.de/algoritmo-en-informatica/. En "informática, un algoritmo es una secuencia de instrucciones secuenciales, gracias al cual pueden llevarse a cabo ciertos procesos y darse respuesta a determinadas necesidades o decisiones. Se trata de conjuntos ordenados y finitos de pasos, que nos permiten resolver un problema o tomar una decisión". 
el árbitro de la ley se pronuncia, pero por ahora, este aspecto no será posible de cuantificar mientras no ocurran los escenarios mencionados.

La interpretación que jueces y juezas hacen sobre las razones que conllevaron a realizar una acción, en el agente racional no puede estimarse, puesto que él resolverá teniendo en cuenta los datos suministrados, con ayuda de los cuales, el camino decisional encontrará soluciones en disciplinas jurídicas. Sin embargo, el trabajo entre jurista y algoritmo se complementa al revisar la iniciativa documental jurídica que se genera con el uso correcto de aquellos aspectos que se entiende, el sistema no puede interpretar, asimilar o aplicar.

\section{ÉTICA DE LA INTELIGENCIA ARTIFICIAL: ENTRE AXIOLOGÍA Y RESPONSABILIDAD}

La ética entendida "como la ciencia o disciplina filosófica que lleva a cabo el análisis del lenguaje moral, [...] la tematización del ethos histórico particular de cada comunidad", ${ }^{31}$ asociado al derecho por medio de la ética judicial, códigos de la profesión en favor del encargado de juzgar; inicia su labor locativa en los jueces digitales, encontrando que no se debe "sobrecargar a los jueces con exigencias sobrehumanas de objetividad e imparcialidad" ${ }^{32}$ ¿Es posible que los sistemas de aprendizaje automático puedan suplir esta petición?

La "ética" así entendida se interesa ante todo por el sentido o la finalidad de la vida humana en su totalidad, se interesa por el bien o el ideal de la vida buena y de la felicidad. [...] En la "ética" se revelan o se encuentran ya dados incluso los fines más elevados que orientan la existencia del hombre o de los miembros de una comunidad en cuanto tales, y a través de los cuales ellos creen poder alcanzar la felicidad o el bien supremo. ${ }^{33}$

La reflexión ética en materia de las innovaciones tecnológicas contemporáneas, en pro del avance de la humanidad, debe ser realizada desde la valoración de criterios axiológicos existentes y su aplicación eficaz en nuevos desarrollos digitales. El reconocimiento de principios éticos judiciales en la normativa de los países ha permitido equilibrar factores sociales en la ley, más allá de una disposición positiva y de obligación, de tal manera que se distingue la validez de la norma con fundamentos.

31. Julio de Zan, La ética, los derechos y la justicia (Montevideo: Fundación Konrad-Adenauer [u.a.], 2004), $19,21$.

32. Ibíd., 108.

33. Ibíd., 22. 
El acelerado desarrollo de la inteligencia artificial ha sido el detonante de profundas transformaciones en numerosos ámbitos de nuestra vida. El poder tecnológico interviene nuestras vidas y las altera debido a su gran fuerza de expansión. La competición entre la inteligencia humana y la inteligencia artificial está servida para las próximas décadas.

Se trata de conocer cuáles son los avances tecnológicos más relevantes en el campo de la IA, y ver qué implicaciones éticas tienen en algunos ámbitos de nuestra vida [...]. Solo si sabemos a qué nos enfrentamos, podremos imaginar alternativas. Es importante asumir responsabilidad desde una heurística del temor, teniendo cautela, no teniendo excesiva confianza, cegados por un tecnocentrismo que puede llegar a esconder un dogmatismo cientificista. ${ }^{34}$

La disciplina en estudio encuentra cimiento en la filosofía de la información. El análisis del dato como suministro primario de la educación de sistemas de aprendizaje automático, emerge como el bien intangible más usado y proporcionado en medio de situaciones de conectividad básicas y elementales de sociedades digitales como las del siglo XXI, donde para poder acceder, ingresar, descargar se debe entregar información sensible con o sin autorización; está última sin duda debe explorarse.

Tal como lo expresan Alchourrón y Bulygin: "El uso primario de lenguaje consiste en comunicar algo a alguien: la comunicación es un acto complejo, en el cual están involucradas por lo menos dos personas: el sujeto activo (el hablante) que emite un mensaje para lo cual usa una fórmula lingüística, y el sujeto pasivo, receptor o destinatario del mensaje". ${ }^{35}$ Asimismo, expresiones de cualquier índole humano-humano o humanomáquina reflejan el tránsito de datos que serán usados en la toma de decisiones.

El aprovechamiento del intercambio comunicacional es el que permite ejecutar procesos donde los sistemas expertos colaboran con los individuos, "la lengua se presenta como un sistema de signos". ${ }^{36}$ Dichos pasos están revestidos de legalidad y legitimidad que respaldan las resoluciones del encargado de juzgar. Por consiguiente, la inteligencia artificial encomendada, de fallar, debe tener unos lineamientos claros en derecho, aplicando justicia enmarcada en un contexto ético que valide socialmente la práctica realizada.

En conjunto con los valores, resalta el principio de responsabilidad. La determinación de quien en calidad de responsable acata y garantiza el ejercicio basado en normas y buenas prácticas, debe estar clarificado dentro de las instituciones o empresas

34. Antonio Luis Terrones Rodríguez, "Inteligencia artificial y ética de la responsabilidad", Cuestiones de Filosofia 4, n. 22 (2018): 144, https://doi:10.19053/01235095.v4.n22.2018.8311.

35. Carlos Alchourrón y Eugenio Bulygin, Sobre la existencia de las normas jurídicas, 1. a reimp, Biblioteca de ética, filosofía del derecho y política, 39 (Ciudad de México: Distribuciones Fontamara, 2002), 14.

36. Paul Ricoeur, El conflicto de las interpretaciones: Ensayos de hermenéutica (Buenos Aires: Fondo de Cultura Económica, 2006), 35. 
promotoras del desarrollo de inteligencia artificial al servicio del derecho. No es una aplicación cualquiera la que se dará, la vida, dignidad, honra, respeto y valor de personas están sujetas a un entrenamiento correcto y legítimo del sistema de aprendizaje automático.

De donde resulta que, se espera que prime la aplicación de principios existentes, trabajados y reconocidos desde los derechos humanos, que servirán de ejemplo para las nuevas implementaciones, sin desconocer la particularidad del caso y ver si valores axiológicos como la responsabilidad existente, abarcan desarrollos en tránsito y venideros. Ocurre que las legislaciones se hacen para proyectos actuales, pero tienen que cubrir futuros avances.

Surge desde este enfoque un nuevo dilema ¿cómo garantizar la eficacia de una norma o catálogo axiológico en aquellos inventos que aún no se idean o se construyen? El reto es ese. No trabajar por cumplir, sino por suplir todos aquellos vacíos legales e ingenieriles que pueden aparecer en el camino de la innovación. Claro, no puede pensarse que todo puede ser previsible, pero sí plantearse temáticas nucleares a regular. ${ }^{37}$

El trabajo en conjunto Estado-legislador-empresa, estará guiado a construir protección de todos los intervinientes y su conservación. En consecuencia, "para la existencia de una norma se requieren, según Von Wright, dos condiciones básicas 1. el dictado de la norma por la autoridad competente y 2. la recepción de la norma por el sujeto normativo". ${ }^{38}$ En definitiva, la ética bien concebida en materia de inteligencia artificial se convierte en un aliado para la validación del sistema como conocimiento artificial judicial. El carácter de autoridad debe revisarse con cuidado, pero como forma puede ser aceptado y válido el juicio artificial racional.

La ética se ata con la verdad. La búsqueda de un sistema experto ético presupone legalidad y veracidad en el procedimiento; así las cosas:

El problema del conocimiento de los futuros contingentes nos lleva al problema de la verdad de las proposiciones acerca de tales acontecimientos. En efecto, el conocimiento supone - entre otras cosas - la verdad: conocer un hecho implica que la proposición que

37. Andrea Martínez Devia, "La inteligencia artificial, el big data y la era digital: ¿una amenaza para los datos personales?", revista La Propiedad Inmaterial, n. ${ }^{\circ} 27$ (25 de junio de 2019): 10-2, https:// doi:10.18601/16571959.n27.01. [...] la Unión Europea ha trabajado en adaptar sus regulaciones a la era digital y la IA, adoptando en diferentes documentos directrices sobre estos temas. Otro de los documentos que expidió la Unión Europea a través de la Comisión Europea en relación con la IA y las nuevas tecnologías fue "El Proyecto de Guía Ética para el Uso Responsable de la Inteligencia Artificial", en la cual, 52 expertos están participando en su elaboración centrándose en el ser humano siempre bajo la luz de la defensa de los derechos fundamentales.

38. Alchourrón y Bulygin, Sobre la existencia de las normas jurídicas, 19. 
lo describe es verdadera. Esto forma parte del concepto de conocimiento y es totalmente independiente de quién sea el sujeto cognoscente. ${ }^{39}$

La conciencia resulta ser un elemento coyuntural. Los personajes centrales del artículo — programadores y jueces - poseen un nivel consciente, propio en la elaboración de sus tareas cotidianas. Sin embargo, para la realización de procesos éticos-legales deben unir fuerzas de manera que, tal como menciona Paul Ricoeur, encuentren una "conciencia de sí, no está en el comienzo sino en el final"; ${ }^{40}$ en otras palabras, el verdadero resultado consciente lo obtendrán en el desenlace de la educación del sistema de aprendizaje automático.

Todo proceso de generación de nuevos asistentes legales está basado en un concepto de conciencia, encontrando su vocación en la conclusión. Así, "Es una idea-límite; la misma que Hegel llamaba 'el saber absoluto'; creamos o no en la posibilidad de decir y articular ese saber absoluto, podemos, en última instancia, ponernos de acuerdo en afirmar que aparece al final, que no es la situación inicial de la conciencia; más aún, es el término de una filosofía del espíritu, no de una filosofía de la conciencia" ${ }^{41}$

Como resultado y expresado lo anterior, un componente primordial en ética y responsabilidad está dado en la determinación explícita de roles como responsables de datos quienes tendrán a su cargo la protección, tratamiento y almacenamiento bajo condiciones estandarizadas que permitan regular, controlar y supervisar la información que alimenta el aprendizaje automático. Además, el cambio de percepción entre ayudante, asistente, colaborador de la inteligencia artificial es necesario para armonizar la convivencia entre individuos y las llamadas máquinas, que se posicionan cada vez más fuerte en mercados mundiales.

\section{DECISIÓN JUDICIAL A TRAVÉS DE INTELIGENCIA ARTIFICIAL: EL ENCUENTRO DE DOS SUBJETIVIDADES}

Las anteriores secciones encuentran su enlace en esta última parte del artículo, puesto que la empatía como factor humano, que expresa emociones, conlleva responsabilidad por parte del encargado en justificar ahora las sentencias. Hallando como actor principal al juez o jueza quien conocedor de la ley interpreta y argumenta, en favor de sustentar el veredicto, siendo un personaje recurrente. Se encargará de la nueva

39. Carlos E. Alchourrón, Eugenio Bulygin y Georg Henrik von Wright, Análisis lógico y derecho, Colección El derecho y la justicia, 24 (Madrid: Centro de Estudios Constitucionales, 1991), 552.

40. Ricoeur, El conflicto de las interpretaciones, 97.

41. Ibíd. 
función sustanciadora el algoritmo, un agente racional, que educado y entrenado con datos jurídicos llegará a concluir en dogmática del derecho.

¿Es a estas subjetividades a las que se refiere el título de la sección? Realmente no, ya que el conocimiento racional implementado puede considerarse $100 \%$ objetivo. Basado en datos de calidad implementados bajo prácticas idóneas ingenieriles, el sistema experto no inmiscuirá en su proceso dictatorial emoción o sentimiento alguno que altere el procesamiento, ¿es esto seguro? El trabajo sigue siendo colaborativo, el raciocinio sigue estando supervisado por el representante del Estado que conoce y aplica la ley.

De allí que, "[1]a figura del juez y la función judicial pueden considerarse como una de las más antiguas de las sociedades humanas. El juez sirve al derecho y a las instituciones, pero la conciencia del derecho no se hizo reflexiva sin la personificación de la Justicia en la figura del juez". ${ }^{42}$ Desde luego, seguirá estando presente en las decisiones como responsable de inicio a fin de procesos judiciales. Son personajes elementales en el proceso actual y en los cambios futuros, del sistema legal de cada país. ${ }^{43} \mathrm{La}$ transición que experimenten los Estados estará atada a su disponibilidad de recursos y compromiso en actualización tecnológica.

Adicional hay que mencionar que, un nuevo actor interviene en el proceso, el configurador informático que conocedor de lenguajes de programación, pondrá su conocimiento en servicio de aplicaciones jurídicas que tendrán la responsabilidad de procesar grandes volúmenes de información en favor de mantener, respetar y aplicar justicia. Esta subjetividad si puede verse involucrada en el desarrollo, de igual manera, en el resultado final. Así que, la revisión de etapas en la consolidación de sistemas expertos decisionales debe ser constante y ¿por qué no?, obligatoria.

Entonces, "[e]s prácticamente redundante señalar que, si hay algo que se espera de los jueces, es que estos sean fundamentalmente justos: pues está a su cargo dirimir conflictos e impartir justicia". ${ }^{44}$ Entonces, ese mismo sentimiento de rectitud debe estar influenciado y arraigado en cada uno de los personajes que tendrán a su cargo la formación, construcción y entrenamiento del nuevo raciocinio artificial. Como apoyo del representante estatal en despachos judiciales se enfatiza en "la relación de concordancia entre una decisión jurídica justificada", ${ }^{45}$ las interpretaciones y fuentes del derecho.

42. Zan, La ética, los derechos y la justicia, 107.

43. Jordi Nieva Fenoll, Inteligencia artificial y proceso judicial, Proceso y derecho (Madrid: Marcial Pons, 2018).

44. Luciana Samamé, "Justicia y empatía: Dificultades y propuestas", Estudios de filosofía práctica e historia de las ideas 18 (2016): 2.

45. José Enrique Sotomayor Trelles, "Emoción, racionalidad y argumentación en la decisión judicial”, Derecho PUCP, n. ${ }^{\circ} 79$ (2017): 152, https://doi:10.18800/derechopucp.201702.008. 
El conflicto puede darse en cuanto al desplazamiento de quien imprime la subjetividad en el fallo. Se cuestionaba anteriormente sobre la autoridad legal, pero aquí se llega a un dilema sobre el desarrollador informático, quien puede llegar a sesgar el algoritmo sin pensarlo, o como herramienta de control que cumpla intereses particulares. Es aquí donde el trabajo que se propone resulta ser colaborativo. Tanto el jurista como el ingeniero entregarán su conocimiento en favor de construir un producto capaz de cumplir con requerimientos de las dos ciencias.

Los grupos deben ser multidisciplinarios, capaces de establecer objetivos claros y que alcancen principios de transparencia, fidelidad y finalidad. Es posible trabajar en favor de obtener altos márgenes de objetividad, no se ha desconocido la influencia por parte de los miembros, pero el compromiso expresado por quienes interactúen en la implementación de proyectos donde la inteligencia artificial tenga un papel relevante, ocasiona que los profesionales se actualicen, optimicen búsquedas, fortalezcan tareas de aprendizaje superior.

Razón por la cual, observar el aporte que trae consigo el uso de tecnologías disruptivas como las estudiadas en este artículo, debe ser aprovechada como el asistente que en realidad es. ${ }^{46}$ Justamente el perfeccionamiento de datos, agrupados en información que se convertirá en conocimiento funge como actividad primordial, y permitirá hacerse toda vez que muchos cálculos y tareas mecánicas serán asumidos por procesadores artificiales, que en busca de permitir al ser humano explorar nuevas alternativas, aparecen para apoyar en el área judicial procesos que requieren toma de decisiones.

\section{CONCLUSIONES}

Desde la perspectiva planteada, puede decirse que la inteligencia artificial alcanza un alto grado de objetividad, pero la variable de aprendizaje a través de los datos puede ser el punto de quiebre en la optimización planteada. Desde luego, los datos

46. Silvia Barona Vilar, "Cuarta revolución industrial (4.0) o ciberindustria en el proceso penal: revolución digital, inteligencia artificial y el camino hacia la robotización de la justicia", Revista Jurídica Digital UANDES 3, n. ${ }^{\circ} 1$ (16 de julio de 2019): 8, https://doi:10.24822/rjduandes.0301.1. "La fascinación por la celeridad y la eficiencia (más con menos) que presenta el mundo jurídico actual han propiciado una transformación de una justicia escrita, lenta, analógica y presencial en un modelo ágil, oral, tecnológico y digital. Y es en esas coordenadas en las que se ha venido favoreciendo la incubación de esta cuarta revolución industrial o lo que se denomina el 4.0 industrial, la Industria Inteligente de la justicia o la Ciberindustria de la justicia del futuro, dado el implacable y penetrante mundo de las TIC en la justicia que nos lleva a plantearnos más allá de su uso instrumental, la posibilidad de que la cuarta revolución industrial (4.0) — nueva manera de organizar los medios de producción - llegue también a la toma de decisiones judiciales y se haga realidad la 'justiciamáquina perfecta"”. 
procesados y convertidos en fragmentos de información serán conocimiento, tanto para el profesional en derecho como el ingeniero y la inteligencia artificial. La forma de ejecutar las acciones ligara estrechamente la responsabilidad individuo-máquinasociedad.

La ética como disciplina es una aliada en cuanto al reconocimiento de la autoridad que puede tener en materia dictatorial el agente racional, así como en el carácter receptivo que tengan los involucrados en el proceso. Por consiguiente, la revisión del catálogo axiológico que interviene en ciclos ingenieriles y jurídicos debe estar atado a un análisis sistémico, de todos aquellos valores que deben y pueden estar presentes al momento de configurar tecnologías disruptivas como las planteadas en este artículo.

Los responsables como figuras educan, conservan y preservan la condición humana. Los procesos de sistemas de inteligencia artificial adquieren un valor esencial en medio de las actividades colaborativas, toda vez que, el desarrollo de actividades cognitivas se convierte en medio que posibilita ampliar conocimiento, permitiendo involucrar principios legales, encaminados a la obtención de justicia.

Las emociones conllevan responsabilidad — sentimiento y acción—, al mencionar que la máquina por esencia y objetivo carece de ellas, ahora las exigencias a nivel de cumplimiento estarán designadas en causas de formación y entrenamiento. Son los personajes designados en crear y educar al sistema experto, quienes deben mantener la objetividad tanto en la aplicación de la norma como en códigos de buenas prácticas ingenieriles.

Para concluir, los agentes racionales asistenciales, educados y entrenados para generar decisiones judiciales plantean optimización de recursos y tiempo a la hora de entregar una sentencia. Son objetivos siempre y cuando juristas y científicos de la información realicen labores de tratamiento de datos óptimos, capaces de impedir que la subjetividad sea impresa por un actor en cualquier fase de la programación. Las emociones como expresión de empatía pueden considerarse parte de dichas particularidades.

\section{BIBLIOGRAFÍA}

Alchourrón, Carlos, y Eugenio Bulygin. Sobre la existencia de las normas jurídicas. 1. ${ }^{\mathrm{a}}$ reimp. Biblioteca de ética, filosofía del derecho y política, 39. Ciudad de México: Distribuciones Fontamara, 2002.

Alchourrón, Carlos E., Eugenio Bulygin y Georg Henrik von Wright. Análisis lógico y derecho. Colección El derecho y la justicia, 24. Madrid: Centro de Estudios Constitucionales, 1991.

Argouse, Aude, y María Eugenia Albornoz, eds. Sentimientos y justicia: coordenadas emotivas en la factura de experiencias judiciales, Chile, 1650-1990. Colección Dossiers, 2. Santiago de Chile: Acto Editores, 2016. 
Barona Vilar, Silvia. "Cuarta revolución industrial (4.0) o ciberindustria en el proceso penal: revolución digital, inteligencia artificial y el camino hacia la robotización de la justicia”. Revista Jurídica Digital UANDES 3, n. ${ }^{\circ} 1$ (16 de julio de 2019): 1-21. https://doi:10.24822/ rjduandes.0301.1.

Campos, Belén. "China y Estonia implantan jueces virtuales para acelerar procesos". El cierre digital: Investigación dirigida a la verdad, 14 de octubre de 2019. https://elcierredigital. com/sucesos/973432190/jueces-virtuales-china-estonia-implantan-algoritmos-agilizantrabajo-judicial.html.

Contreras Ugarte, Jesús Víctor Alfredo. "Cimentando en valores para empoderar en derechos y al derecho. Empatía del reconocimiento. Una reflexión desde la filosofía de Hegel”. Universitas. Revista de Filosofía, Derecho y Política, n. ${ }^{\circ} 23$ (2016): 159-91.

Diccionario Sensagent. "Ginoide". Accedido 12 de octubre de 2020. http://diccionario.sensagent.com/Ginoide/es-es/.

González de Rivera y Revuelta, José Luis. “Empatía y ecpatía”. Psiquis 25, n. ${ }^{\circ} 6$ (2004): 243-5.

González Lagier, Daniel. Emociones, responsabilidad y derecho. Colección Filosofía y derecho. Madrid: Marcial Pons, 2009.

Herbas, Graciela Noelia. "La sensibilidad humana en el profesional del derecho como instrumento esencial para una efectiva justicia". Revista Derecho y Salud (2018): 98-110.

IBM. "Machine Learning", 22 de junio de 2020. https://ibm.com/ar-es/analytics/machinelearning.

James, William. “¿Qué es una emoción?”. Traducido por Elena Gaviria Stewart. Estudios de Psicología, n. 21 (1985): 57-73.

Martínez Devia, Andrea. "La inteligencia artificial, el big data y la era digital: ¿una amenaza para los datos personales?”. Revista La Propiedad Inmaterial, n. 27 (2019): 5-23. https:// doi:10.18601/16571959.n27.01.

Monjaraz Fuentes, Paulina. "Reconstruir la comunidad humana desde la empatía: Medios para generar la paz". Open Insight X, n. ${ }^{\circ} 20$ (2019): 79-94.

Montañez Sierra, Cristian Fernando. "Decisiones judiciales asistidas: Paradigmas del juez y jueza en cuanto al uso de inteligencia artificial”. Tesis de maestría de próxima aparición, Universidad Andina Simón Bolívar, Sede Ecuador, s. f.

Nieva Fenoll, Jordi. Inteligencia artificial y proceso judicial. Proceso y derecho. Madrid: Marcial Pons, 2018.

Nussbaum, Martha. La ira y el perdón: Resentimiento, generosidad, justicia. Traducido por Víctor Altamirano. Ciudad de México: Fondo de Cultura Económica, 2018.

- Las emociones políticas: ¿Por qué el amor es importante para la justicia? Traducido por Albino Santos Mosquera. Barcelona: Paidós, 2014.

Peña Valenzuela, Daniel. “Inteligencia artificial y derecho”. Presentado en Seminario IV: Inteligencia artificial, Universidad Externado de Colombia, 2019.

Raffino, María Estela. “Algoritmo en informática". Concepto.de. Accedido 12 de octubre de 2020. https://concepto.de/algoritmo-en-informatica/. 
Revista de Robots. "Androides, qué es un androide humano y ejemplos". Revista de Robots, 30 de enero de 2020. https://revistaderobots.com/robots-y-robotica/androides-que-es-unandroide-humano-y-ejemplos/.

Ricoeur, Paul. El conflicto de las interpretaciones: Ensayos de hermenéutica. Buenos Aires: Fondo de Cultura Económica, 2006.

Samamé, Luciana. “Justicia y empatía: Dificultades y propuestas”. Estudios de filosofía práctica e historia de las ideas 18 (2016): 1-12.

Smith, Adam, y Carlos Rodríguez Braun. La teoría de los sentimientos morales. Madrid: Alianza, 1997.

Sotomayor Trelles, José Enrique. "Emoción, racionalidad y argumentación en la decisión judicial”. Derecho PUCP, n. 79 (2017): 151-90. https://doi:10.18800/derechopucp.201702.008.

Sulayes, Antonio Rico. "Hacia el reconocimiento de la subjetividad en los agentes artificiales. Una delimitación del sujeto artificial en la semiótica”. Tópicos del Seminario 41 (2019): 147-69.

Terrones Rodríguez, Antonio Luis. "Inteligencia artificial y ética de la responsabilidad". Cuestiones de Filosofía 4, n. ${ }^{\circ} 22$ (7 de agosto de 2018). https://doi:10.19053/01235095. v4.n22.2018.8311.

Tripero, Tomás de Andrés. "Inteligencia emocional artificial: En las fronteras de la robótica". Universidad Complutense de Madrid, s.f.

Wasserman, Moisés. “Empatía”. ProQuest, 24 de enero de 2020. https://proquest.com/docvie $\mathrm{w} / 2344144141$ ? accountid $=8308$.

Zan, Julio de. La ética, los derechos y la justicia. Montevideo: Fundación Konrad-Adenauer [u.a.], 2004. 